PDFlib PLOP: PDF Linearization, Optimization, Protection

Page inserted by evaluation version www.pdflib.com - sales@pdflib.com 


\title{
Executive Summary: The Emergency Neurologic Clinical Trials Network Meeting-A National Institute of Neurological Disorders and Stroke Symposium
}

\author{
Arthur M. Pancioli, MD, William G. Barsan, MD, Robin A. Conwit, MD
}

Many neurologic conditions present emergently, and patient outcomes can often be determined by the care provided in the first hours after onset. Some of these conditions occur frequently (stroke, traumatic brain injury, seizures) and are associated with considerable morbidity and costs, and some occur less frequently (spinal cord injury) but have very high morbidity and high costs associated with their care. Most of the clinical research in the past has focused on chronic and subacute care, and there is often limited evidence to support the acute management of many of these conditions. Multicenter research networks have typically been designed to answer disease-specific questions and have not been organized to address other neurologic conditions that may also be present in the patient population. Each time a new clinical question is addressed, a new clinical research network is established and is usually terminated after the clinical question is answered. Significant start-up costs are associated with the establishment of these networks and are iterative in each new study.

On March 17 and 18, 2004, the National Institute of Neurological Disorders and Stroke (NINDS) sponsored a conference to explore the advisability of establishing a multicenter network designed to perform clinical trials in emergent neurologic conditions. The Emergency Neurologic Clinical Trials Network (ENCTN) concept was discussed by 25 clinicians and scientists from multiple disciplines. The goal was to improve the overall functional outcome for patients with acute neurologic emergencies. The participants discussed various aspects necessary in evaluating the potential of such a network, including the orga-

From the Steering Committee, University of Cincinnati (AMP), Cincinnati, OH; Steering Committee, University of Michigan (WGB), Ann Arbor, MI; and National Institute of Neurological Disorders and Stroke, National Institutes of Health (RAC), Bethesda, MD.

Received June 9, 2004; accepted June 14, 2004.

This article may be published concurrently in Annals of Emergency Medicine.

Address for correspondence and reprints: Arthur M. Pancioli, MD, University of Cincinnati, Department of Emergency Medicine, 231 Albert Sabin Way, Cincinnati, OH; e-mail: arthur.pancioli@uc.edu. doi:10.1197/j.aem.2004.06.009 nizational structure, funding, cost-effectiveness, and clinical conditions to be studied. A neurologic emergencies network that is not disease-specific would open opportunities for clinical research that would facilitate rapid and effective treatment of emergent conditions and lead to improved patient outcomes. In addition, the cost savings realized through economies of scale of such a network would allow more research to be performed at a lower cost. The network would have at least three clinical trials selected at the time of network activation, which could be either phase II or phase III clinical trials. To maintain the network and take advantage of economies of scale, new projects would be continuously developed. The conference participants identified multiple potential clinical trials that could be performed by the network and answer important clinical questions. By facilitating highpriority, interdisciplinary, multi-institutional research into the diagnosis and treatment of neurologic emergencies, such a network will ultimately lead to new therapies for our patients. The full report is available on the Web at www.ninds.nih.gov/news_and_events / proceedings/enctn_workshop.htm.

\section{EXECUTIVE SUMMARY}

Neurologic emergencies constitute a number of serious conditions leading to significant morbidity and mortality yearly in the United States. Many of these conditions are relatively uncommon, and there is a paucity of evidence from randomized trials to support clinical decisions, particularly in the emergency setting. Neurologists and neurosurgeons are not present during the emergency presentations of many of these disorders, and traditional neurologic research has seldom addressed these conditions in the out-ofhospital or emergency department settings.

One significant barrier to performing the necessary studies of many neurologic emergencies is the low frequency of presentation to any given institution. Therefore, single institutions or small groups of institutions lack patients for adequately powered studies. A second significant barrier is the tremendous time and resources required to establish networks for multicenter 
trials. Prior studies of acute neurologic conditions (e.g., the NINDS study of tissue-type plasminogen activator in stroke) required the development of unique multicenter groups that disbanded after the study. New studies then require new investigators to construct new networks.

One solution to both of these problems is to create a large, scalable network of institutions capable of performing research on multiple different acute neurologic emergencies. Thus, multiple relatively uncommon processes that present as acute neurologic emergencies could be studied simultaneously, achieving substantial economies of scale and allowing for adequate sample sizes. The heavy start-up costs for the network would be incurred only once, and support for each of the individual studies would correspondingly be decreased.

It is the intent of this article to propose the concept of a large, scalable network capable of performing clinical research on acute neurologic emergencies. In order to do so, a group of clinical investigators came together to evaluate this concept and generate a potential model of such a network. The following is an outline for the process of evaluation and potential design for such a network.

1. Define the mission.

2. Define the scope.

3. Design the governance and access.

4. Design the structure.

5. Describe the type of studies to be performed by this network.

6. Evaluate the financial implications of such a network.

7. Evaluate the potential data-sharing structure of the network.

8. Define the necessary human subject protection for all potential patients.

Mission. The mission of the ENCTN is to optimize care and improve outcomes for patients who seek care in emergency departments for acute neurologic disorders. The ENCTN will achieve its mission by facilitating high-priority, interdisciplinary, multi-institutional research in the diagnosis and treatment of neurologic emergencies.

Scope. The ENCTN will encompass a broad range of high-morbidity and high-mortality conditions for which treatment outcomes are poor or for which little rigorous evidence guides treatment decisions. This network will be built on a "hub-and-spoke" model. This will be a scalable network of multiple hubs, each responsible for multiple spokes.

Governance and Access. The ENCTN should act in a manner similar to a public utility. It must have clearly defined governance and access. It must be designed to foster a broad range of investigations and to ensure that the investigations meet the highest scientific standards. Participation in the research by both academic and private practitioners in multiple disciplines will foster acceptance of results to redefine the standard of care. The ENCTN should be open to investigator-initiated proposals as well as those from industry.

Essential Elements in the Governance of a Clinical Trial Network. Most successful clinical trials networks include a clinical coordinating center and a data coordinating center. An executive or steering committee governs a series of subcommittees with charges ranging from protocol review to publications.

The ENCTN steering committee, built on this successful "board of directors model," would have a chairperson, likely the director of the clinical coordinating center, and other critical members, including a predetermined number of principal investigators from the hubs who rotate on and off the steering committee at preset intervals. Also included are the director of the data coordinating center, one or more members from the NINDS, and the chairs of each subcommittee.

The ENCTN will require a manual of operations to guide the steering committee and subcommittees. The organization will require a geographic home. This could house the clinical coordinating center or the data coordinating center or both, although these may be at separate locations.

Access and Relationship to Funding (National Institutes of Health and Industry). Studies to be performed within this network can enter the network via at least two routes.

One route is through investigator-initiated studies, which would be reviewed by an ad hoc review group or study section. This review group/study section would include members from the executive committee of the network. This would ensure peer review from experts in the specific disease state as well as input from administrators with knowledge of the network structure and capabilities.

Studies to be funded by nongovernmental entities such as industry can be proposed directly to the executive committee. The inclusion of NINDS staff on the executive committee ensures that the infrastructure being funded by the National Institutes of Health/ NINDS provides the appropriate scientific return on the investment.

Who Are the People and Institutions in the Network? Given the breadth of projects that the ENCTN could potentially conduct, a model for the ENCTN network must provide great flexibility, scalability, and institutional breadth. An essential ingredient will be emergency medicine and neurology/neurosurgery collaboration in the participating 
institutions. In many institutions, this will represent a significant paradigm shift, because there has not been a long history of close ties between these specialties. Fostering the development of collaboration between emergency and neurologic specialists will be an essential task for the clinical coordinating center.

To enable a scalable network, a hub-and-spoke model is being proposed. The hubs will be the backbone of the network, and all clinical projects will be conducted at the hub centers. For studies requiring larger sample sizes, the network can be "scaled up" by the addition of spokes. Regional hubs will provide research and clinical infrastructure for the spokes (i.e., nearby collaborating hospitals with investigators) but perhaps without full-time research staff or advanced care capabilities. A hub is not synonymous with the coordinating center. The network will be composed of dozens of hubs each supporting, on average, two to five regional spoke institutions. Hubs will likely be regional academic medical centers, the local tertiary care facilities in the region.

Spokes will vary in institutional format and will include academic centers, community hospitals with academic affiliation, and community hospitals. Incorporating spokes that are not based at tertiary care centers will increase enrollment and expand patient access to studies while allowing evaluation of the study intervention in a real-world practice setting, providing an estimate of the intervention's clinical effectiveness. Depending on the complexity of the study, spokes may either enroll study patients locally, utilizing the hub only for general guidance, or may identify and enroll patients at the spoke before transfer to the hub for specialty care. Unlike many hub-and-spoke models, the ENCTN should also include local practice-based neurologists. These specialists would provide a referral base for potential patients and would serve as coinvestigators supporting longitudinal outcome evaluations.

Participants in each spoke will vary by study. The core members at each institution will be emergency physicians with neurologic collaboration. Given the existing shortages of specialty physicians, not all centers will have in-house access to neurologists or neurosurgeons. In these settings, access to the hub's neurologic expertise may be sufficient. In more complex therapeutic studies, lack of neurologist or neurosurgeon specialists will mandate transfer to hub institutions for continuance of the study.

Each hub and spoke will require various support staff commensurate with the studies being conducted at each institution. To some degree, a research nurse or research coordinator should be identified and funded to support the ENCTN efforts at each institution. The level of training and commitment will vary by institution.

What Are the Topics for Research, and How Will They Be Determined? The ENCTN should augment the efficiency and productivity of the nation's clinical research enterprise. It should provide the capability to conduct, more rapidly and efficiently, multiple highquality clinical studies and trials in emergency neurology. The full report contains sections addressing critical issues related to 1) the research agenda of the ENCTN; 2) the possible mechanisms for targeting and prioritizing specific neurologic conditions for the ENCTN; 3) possible mechanisms for submitting, soliciting, and reviewing proposals for this network; and 4) initial research questions that the ENCTN would be ideally and uniquely positioned to perform.

Research Agenda of the ENCTN. In general terms, the mission of the ENCTN is to optimize care and improve outcomes of patients who seek care in emergency departments for acute events affecting the nervous system. The ultimate measure of success for clinical trials is the reduction of mortality and disability in persons who present to the emergency department after experiencing acute neurologic events.

In specific terms, the research agenda of the ENCTN will involve two categories of diagnoses. The first category comprises high-prevalence neurologic diagnoses such as ischemic stroke. Despite the high prevalence of these neurologic diagnoses, many diagnostic and therapeutic questions are unanswered. Treatment protocols vary widely among emergency departments, and little evidence supports current diagnostic and therapeutic practices. Clinical trials demonstrating even modest treatment effects for these high-prevalence diagnoses would result in large absolute benefit in reduction of human suffering and economic losses. Accurately estimating critical, although small, treatment effects requires large absolute numbers of study participants. The ENCTN would have the large number of centers required to perform this research. The involvement of a large number of centers should also greatly enhance dissemination of research findings into the community, accelerating the pace at which new, effective treatments improve outcomes nationally.

The second category is low-prevalence but highmorbidity and high-mortality neurologic diagnoses. These diagnoses are not seen at single centers in sufficient numbers to allow adequately powered clinical trials. Yet, from a standpoint of human suffering and societal burden, they merit careful clinical trials. Much clinical research to date on these problems has been piecemeal and inconclusive. For these lower-prevalence diagnoses, even multicenter trials can fall short of necessary sample sizes. Thus, the ENCTN would be ideally suited to involve the large number of hospitals, both university- and community-based, required to enroll sufficient numbers of patients. Examples of high- and low-prevalence neurologic diagnoses are shown in Table 1. 
TABLE 1. Examples of High- and Low-Prevalence Neurologic Diagnoses

\begin{tabular}{ll}
\hline $\begin{array}{c}\text { High-Prevalence } \\
\text { Neurologic Diagnoses }\end{array}$ & $\begin{array}{c}\text { Low-Prevalence, } \\
\text { High-Morbidity/Mortality } \\
\text { Neurologic Diagnoses }\end{array}$ \\
\hline Stroke & Global brain ischemia \\
Intracerebral hemorrhage & Venous sinus thrombosis \\
Subarachnoid hemorrhage & Spinal cord injury \\
Traumatic brain injury & Meningitis/encephalitis \\
Seizure/status epilepticus & \\
\hline
\end{tabular}

Criteria for Targeting and Prioritizing Specific Neurologic Conditions for ENCTN Study. Proposals for clinical trials through the ENCTN should be evaluated for significance to human health and scientific merit. With regard to significance to human health, we propose these factors to be considered in prioritizing clinical studies and trials:

1. Prevalence of the condition

2. Mortality

3. Morbidity, including lost quality-adjusted life years

4. Economic impact

5. Relevance to the National Institutes of Health Roadmap goals and appropriateness for ENCTN.

Other Research Considerations. While the main thrust of the ENCTN will be interventional studies, there will be an excellent opportunity to incorporate observational studies into the larger context of these interventional trials. The ability of the network to collect observational data will be of critical importance in evaluating new areas for interventional studies and providing data regarding numbers of patients and outcomes with conventional therapy before designing interventional studies.

The ENCTN will be best utilized if there are multiple simultaneous studies in different disease areas. The simultaneous conduct of multiple studies will enable greater economies of scale, and the projects can be phased in such a way that there is no "idling time" present in the network.

Is the Research More Cost-effective If Done by the Networks? The cost analysis in the full report is organized into three subsections. In the first subsection, a simplified theoretical framework for identifying and calculating the major components of cost is described. In the second subsection, we consider the factors that may make a network a more efficient strategy for setting up new multicenter clinical trials. In the final section, we use this framework to create an approximate estimate of the relative steady-state costs of the ENCTN.

The task force determined that beyond the potential value of developing the ENCTN in promoting the social welfare, we estimate that developing such a network would make economic sense. Specifically, for a fixed portfolio of three large clinical trials, we estimate that for an initial investment of approximately $\$ 1.5$ million, a network would result in a net savings of nearly $\$ 8$ million. This savings would result primarily from efficiencies in the operation of trials, because modest decreases in per-subject costs are multiplied over a large number of subjects. To predict more precisely the cost impact of research performed in the ENCTN, it would be essential to specify the projects in some detail. Despite the highly speculative nature of the current estimates, they do suggest that the ENCTN could be economically feasible.

How Can Data Management Be Handled Most Efficiently? To facilitate multicenter clinical trials in emergency neurologic conditions, it is critical that data be collected in an efficient, cost-effective, and readily accessible manner that facilitates merging data from a variety of sources and takes into consideration current Health Insurance Portability and Accountability Act (HIPAA) compliance issues. The full report of this task force addresses critical issues related to 1) the type of data entry forms, 2) enrolling and tracking patients, 3) merging data from a variety of sources, 4) centralized data safety monitoring, 5) protocol compliance, 6) the need to generate regular (e.g., monthly) data reports, and 7) compliance with current HIPAA regulations. Based on current advances in Web-based technologies, electronic data forms, and availability and access to the Internet, it is apparent that the management of the ENCTN would be greatly facilitated by an efficient Web-based data management system.

To maximize the efficiency of the ENCTN and the quality of collected data, a centralized electronic data collection strategy is required. The use of Web-based approaches has several advantages over non-Webbased systems. The capability to incorporate complex data from a variety of sources will need to be built in. This approach could facilitate the activities of a data safety monitoring board. HIPAA compliance issues are important to consider when designing the centralized data collection strategy. Several examples of successful application of Web-based strategies to run clinical trials have been reported in the literature and attest to the utility and practicality of this approach.

What Are the Human Subjects Research Issues? While there are many ethical aspects to research, the ENCTN as a whole will most likely be concerned with those related to the rights of human subjects in research, especially those made vulnerable by a devastating disease or injury. These include, but are not limited to, the issues of meaningful consent for research participation, privacy of medical records, and adequate recruitment of minority patients into clinical trials. There are also unique aspects of clinical trials 
research and out-of-hospital research that pose special challenges for emergency neurology researchers.

Issues associated with informed consent and privacy will be common for all centers participating in the network. The goals of the ENCTN will be to inform and educate patients and to protect patient privacy. Site investigators will need to be aware of local and state regulations. The ENCTN will work with local sites to enhance patient protections as well as patient recruitment and enrollment. An expert panel, developed from within and outside of the ENCTN, will advise investigators on ethical, privacy, and access issues.

\section{SUMMARY}

The NINDS conference brought together a diverse group of clinicians/scientists to evaluate the advisability and feasibility of establishing a network to conduct clinical trials in emergent neurologic conditions. The attendees developed a structural framework for the network and identified potential clinical trials to be performed in the network. The network would be built on a hub-and-spoke model and would incorporate academic as well as community hospitals, leading to better generalizability of the research findings to the community at large. The conduct of multiple simultaneous clinical trials in the network should lead to economies of scale and reduced costs in performing clinical research. Establishment of a clinical research network has the potential to significantly impact the morbidity and mortality from acute neurologic disorders and lead to improved outcomes and cost savings in the future.

The authors are grateful to the many outstanding and knowledgeable individuals from a variety of professions and medical specialties who contributed to this conference. 